\title{
Aplicativo colaborativo para alerta de vulnerabilidade a alagamentos e enchentes no Vale do Itajaí
}

\author{
Andressa S. A. de Lima ${ }^{1}$, Ana Elisa F. Schmidt ${ }^{1}$, Rodrigo R. Nogueira ${ }^{1}$, Paulo Ce- \\ sar F. de Oliveira ${ }^{1}$ \\ ${ }^{1}$ Instituto Federal Catarinense - Campus Camboriú (IFC) \\ Caixa Postal 2016 - 88340-055 - Camboriú - SC - Brasil \\ andressasts1@gmail.com, \\ \{ana.schmidt, rodrigo.nogueira,paulo.oliveira\}@ifc.edu.br
}

\begin{abstract}
Santa Catarina is one of the states that are more affected by the results of flooding in Brazil, which compromises public health and quality of life. Acknowledging this reality, this article introduces a software that allows users to alert about the danger of that kind of natural disaster in the cities of Vale do Itajai. This paper presents the design and modeling of an Android's mobile application that helps residents with the warning and knowledge about the risk of such disasters. The application is developed with the Apache Cordova platform, Javascript, HTML5 and CSS3 languages and the implementation of a map on its screen using the Google Maps Application Programming Interface.
\end{abstract}

Resumo. Santa Catarina é um dos estados que mais sofre com alagamentos e enchentes no Brasil, o que como consequência compromete a saúde e qualidade de vida pública. Reconhecendo esta realidade, este artigo apresenta um software que permite aos usuários o alerta desse tipo de desastre nas cidades do Vale do Itajaí. Este artigo descreve o design e modelagem de uma aplicação para dispositivos móveis Android que auxilia os moradores com o alerta e conhecimento sobre o risco desse tipo de desastre. A aplicação é desenvolvida com a plataforma Apache Cordova, as linguagens Javascript, HTML5 e CSS3, com a implementação de um mapa através da Interface de Programação de Aplicativos do Google Maps.

\section{Introdução}

Os alagamentos são um grande problema que afeta uma parte significativa da população de Santa Catarina. Com isto, faz-se necessário pôr à disposição dos moradores das áreas vulneráveis meios mais abrangentes e acessíveis para alerta dos desastres, destacando a importância do aviso às autoridades.

As enchentes são desastres decorrentes de ações naturais ou antrópicas que geram graves consequências e derivam das enxurradas. Um exemplo de causa natural como fator são períodos de intensidade de chuvas. Já os alagamentos, diferente das enchentes, são acúmulos das águas da chuva em áreas impermeabilizáveis [Licco e Mac Dowell, 2015]. Além do descaso ambiental, esses tipos de desastres se associam a problemas de saúde pública, já que promovem a proliferação e contaminação de doenças graves, como dengue, hepatite A e leptospirose, que são transmitidas pela mistura da água com o esgoto ou lixo humano [Cunha, 2016]. 
Considerando o impacto dessas situações de calamidade pública na vida da população que habita áreas de risco, este artigo apresenta a modelagem de um aplicativo colaborativo para dispositivos móveis com Android que leve à população informações de alerta de alagamentos e enchentes que ocorrem em sua região. Esse aplicativo utiliza-se da informação fornecida pelos usuários das áreas atingidas para estabelecer a situação de uma região, de acordo com os conhecimentos e experiências dos mesmos. O aplicativo também informa e orienta os usuários sobre as providências a serem tomadas nessas situações, em telas do aplicativo que podem ser acessadas a qualquer momento.

Também cabe destacar que o uso de aplicativos para dispositivos móveis pode tornar-se muito útil nessas situações, já que, segundo a Fundação Getúlio Vargas, o Brasil tem atualmente 280 milhões de dispositivos móveis conectáveis à internet [Brigatto, 2017].

\section{Metodologia}

Esse trabalho é categorizado como uma pesquisa aplicada [Marconi e Lakatos, 2010] e iniciou-se com a elaboração do repertório conceitual, onde foi realizada pesquisa exploratória, bibliográfica e documental, através artigos e reportagens on-line.

O aplicativo trata-se de uma aplicação híbrida, tipo que agrega as tecnologias de desenvolvimento e programação de aplicações nativas e web com as linguagens de marcação de hipertexto HTML5 (HyperText Markup Language) e folhas de estilo em cascata, o CSS (Cascading Style Sheets) [Giroldo e Fressati, 2015], e Javascript para o desenvolvimento do aplicativo móvel [Piran e Lazzaretti, 2015]. Esse tipo de desenvolvimento de aplicações móveis a ser empregado no projeto é feito através do Apache Cordova.

Para implementar o mapa e suas funcionalidades está sendo utilizada a Interface de Programação de Aplicativos do Google Maps, que proporciona a manipulação de pontos no mapa e delimitação de regiões, inserção de conteúdo, dentre outros [Fernandes, 2009].

A modelagem do aplicativo iniciou-se com a delimitação do seu escopo, seguido da definição do perfil dos usuários e do levantamento de requisitos. Foi realizada a modelagem da base de dados do sistema e, por fim, o desenvolvimento dos protótipos da interface do aplicativo. Com o processo de modelagem do sistema foi procurado adaptar sua usabilidade para o conhecimento e experiência do usuário, considerando a forma que essa recorreria ao sistema para tratar de situações de perigo.

\section{Modelagem do Aplicativo}

O funcionamento do aplicativo pretende ser simples e intuitivo e sua interface apresenta um mapa para situar o usuário e fazer com que esse consiga visualizar as localizações dos bairros onde é apresentada sua condição em relação a possibilidade de enchentes ou alagamentos.

Para alcançar tal objetivo, os requisitos funcionais foram definidos e na Tabela 1 são apresentados alguns dos principais requisitos funcionais, identificados como RF, elencados. Esses requisitos foram elaborados tendo em vista os diferentes tipos de usuários que vão utilizar o aplicativo e seus respectivos papéis. Apesar de não listados na tabela abaixo, durante a etapa de modelagem também foram elencados os requisitos não funcionais e regras de negócio do aplicativo. 
Tabela 1 - Principais requisitos funcionais do aplicativo. Fonte: Autores.

\begin{tabular}{l|l}
\hline RF & Descrição do requisito funcional \\
\hline RF01 & Cadastrar as informações do usuário; \\
\hline RF02 & $\begin{array}{l}\text { Para cada bairro destacado no mapa, em seu ícone de localização deve-se } \\
\text { apresentar na tela sua condição em relação à vulnerabilidade a desastres; }\end{array}$ \\
\hline RF03 & $\begin{array}{l}\text { O usuário deve poder alertar que seu bairro se encontra em perigo desses } \\
\text { desastres; }\end{array}$ \\
\hline RF04 & As informações do sistema devem ser salvas em um banco de dados; \\
\hline RF05 & $\begin{array}{l}\text { O sistema deve enviar um e-mail ao usuário quando muitas pessoas alertarem } \\
\text { sobre perigo em seu bairro; }\end{array}$ \\
\hline RF06 & $\begin{array}{l}\text { O sistema deve apresentar, em forma de lista, a relação de cidades e seus } \\
\text { bairros e como eles se encontram; }\end{array}$ \\
\hline RF07 & $\begin{array}{l}\text { O marcador do bairro deve ser apresentado no mapa em conjunto com o } \\
\text { nome do bairro e sua condição. }\end{array}$ \\
\hline
\end{tabular}

Foram desenvolvidos protótipos de média fidelidade da interface do aplicativo, sendo que na Figura 1(a) é mostrada a tela do aplicativo com o mapa da região e seus bairros; já na Figura 1(b) é mostrada a tela de apresentação da situação de um bairro em condição alarmante, e na Figura 1(c) é apresentada a interface do aplicativo com a relação de bairros e suas condições atuais.

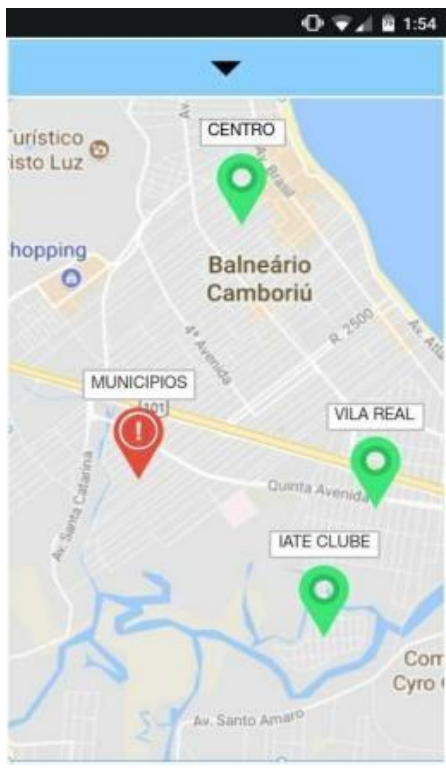

(a)

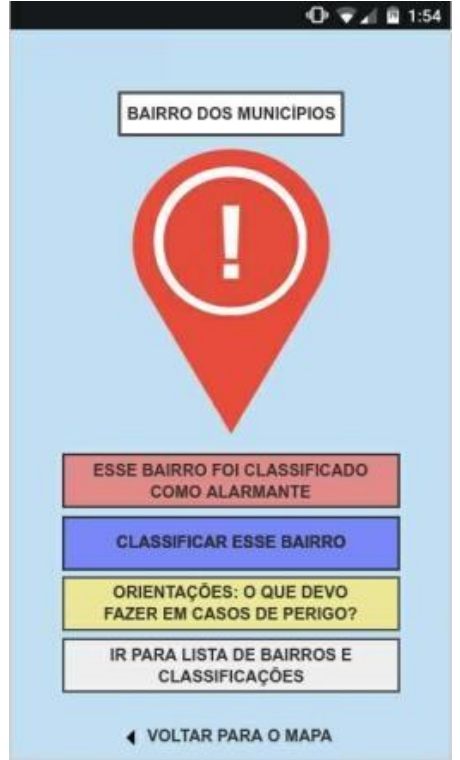

(b)

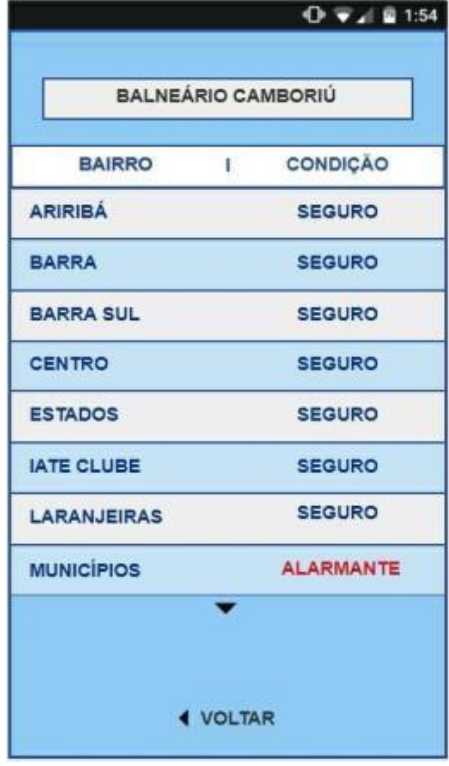

(c)

Figura 1. Protótipos da interface do aplicativo. Fonte: Autores.

Também foi realizada a modelagem do banco de dados a ser implementado na aplicação. É utilizado o SGBDR, Sistema Gerenciador de Banco de Dados, PostgreSQL e a SQL, Structured Query Language, para a criação, consulta e manipulação da base de dados. Na Figura 2 é mostrado o modelo lógico do banco de dados do aplicativo. 


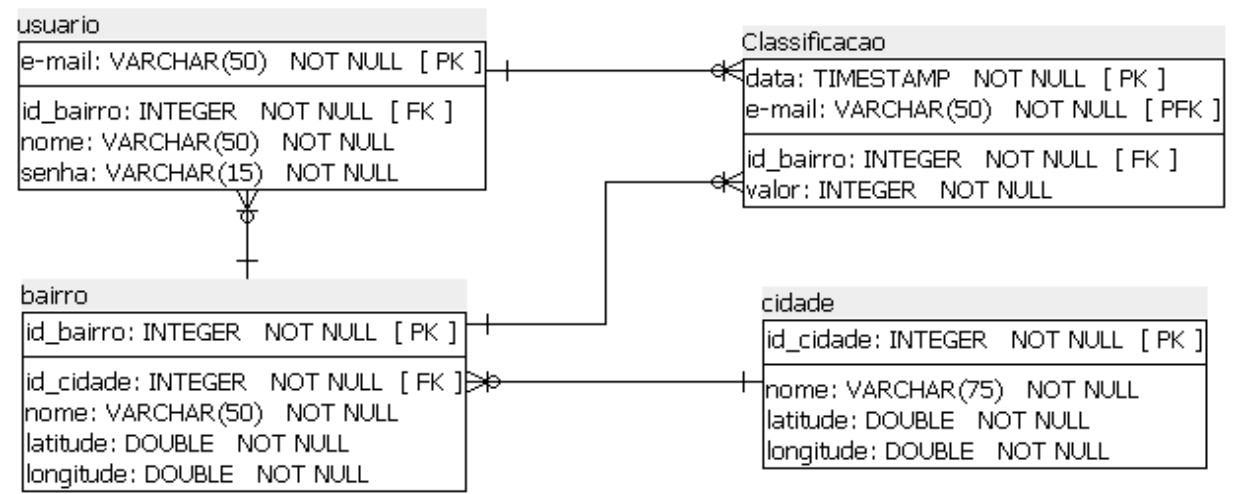

Figura 2. Modelo lógico do banco de dados do aplicativo. Fonte: Autores.

\section{Considerações Finais}

A modelagem desse aplicativo destaca-se por agregar os conhecimentos de diversas disciplinas presentes no curso Técnico em Informática integrado ao Ensino Médio, como Programação e Desenvolvimento Web, Design Gráfico, Banco de Dados e Geografia.

Atualmente o aplicativo encontra-se em fase de implementação e deve estar concluído ao final de 2018. Espera-se que o mesmo sirva como uma nova forma de dar assistência à população em situações de desastres como alagamentos e enchentes.

\section{Referências}

Brigatto, Gustavo. (2017). "Brasil terá um smartphone por habitante até outubro, projeta FGV”, In: Valor Econômico on-line. Disponível em: http://www.valor.com.br/empresas/4943034/brasil-tera-um-smartphone-por-habitante-ate-outubro-projeta-fgv.

Cunha, J. (2016). "Enchentes em São Paulo aumentam riscos de leptospirose e hepatite". Disponível em: http://www1.folha.uol.com.br/cotidiano/2016/03/1748792-enchentesem- sao-paulo-aumentam-riscos-de-leptospirose-diarreia-e-hepatite-a.shtml.

Fernandes, P. (2009). "Entendendo um pouco a API Google Maps". Disponível em: https://imasters.com.br/artigo/13893/apis-google/entendendo-um-pouco-a-api-google- $\operatorname{maps} /$ ?trace $=1519021197 \&$ source $=$ single .

Giroldo, B. e Fressati, W. (2015). "Evolução no desenvolvimento com HTML5”. Disponível em: http://web.unipar.br/ seinpar/2015/_include/artigos/Bruno_Chesine_Giroldo.pdf.

Licco, E. A. e Mac Dowell, S. F. M. (2009). "Alagamentos, Enchentes Enxurradas e Inundações: Digressões sobre seus impactos sócio econômicos e governança”. Disponível em: http://www.sp.senac.br/blogs/revistainiciacao/wp- content/uploads/2015/12/110_IC_artigo-.pdf.

Marconi, M.A.; Lakatos E. M. (2010). Técnicas de pesquisa: planejamento e execução de pesquisas, amostragens e técnicas de pesquisas, elaboração e interpretação de dados. São Paulo: Atlas.

Piran, F. M. e Lazzaretti, A. T. (2015). "Estudo da Tecnologia PhoneGap/Cordova e a aplicação em um estudo de caso". Disponível em: http://painel.passofundo.ifsul.edu.br/uploads/arq/20160331164543877842707.pdf. 